\title{
Towards a theory of electromagnetic effects arising in acoustically excited electrolyte solutions
}

\author{
Alexander V. Kramarenko ${ }^{1}$, Andrey V. Kramarenko ${ }^{2}$, and Oksana Savenko ${ }^{3}$ \\ ${ }^{1}$ TREDEX Company Ltd, PO box 11515, Kharkiv, Ukraine 61001, http://www.tredex-company.com/en, \\ mailto: tredexcompany37@gmail.com \\ ${ }^{2}$ General and Inorganic Chemistry Department, National Technical University "KhPI", 2 Kyrpychova Str., \\ Kharkiv, Ukraine 61002, mailto: andrii.kramarenko@khpi.edu.ua \\ ${ }^{3}$ School of Radiophysics, Biomedical Electronics and Computer Systems, Karazin Kharkiv National \\ University, 4 Svobody Sq., Kharkiv, Ukraine 61022, mailto: xana.savenko@gmail.com
}

\begin{abstract}
The present work is an effort to explain theoretically the physics of some processes we have observed in our previous experiments. They occur under any mechanical excitation in solutions of strong electrolytes. We assume that the occurrence of the low-frequency Debye ionic vibration potential (IVP) and the deviation of the RF polarization vector are conjugated, but only in the sense that the power flux density of some physical process " $\mathrm{X}$ " responsible for the rotation of the polarization vector is proportional to the square of the electric potential voltage. While the independence of the RF anisotropy appearance from the applied voltage and from the Debye potential in particular has been proved experimentally. An equivalent electrical circuit that simulates the observed effects within the solution excited by an acoustic wave is proposed and tested for physical feasibility. Special attention is paid to the basic theory of the ionic vibrational potential, namely, its predictions in the low-frequency range, which contradict both experiment and the energy conservation law. Given the futility of describing the "memory" effect as a process of electrical or molecular origin, several arguments are presented in favor of the fluidgyroscopic mechanism. It was suggested that the rotation of the polarization vector of the RF signal is due to a change in the electric moment of the liquid atoms and/or the nuclear moment of ions having an odd mass number. The applications of the research are also supplemented. The results of new experiments show that the RF anisotropy of the solution is transported by the carrier. Accordingly, it is possible to create a completely contactless unitary sensor of velocity and inhomogeneities of the liquid, moreover, the experimental setup has previously confirmed the affordability of the idea.
\end{abstract}

In the previous article 1], we couldn't formulate any complete theoretical foundations of the discovered physical effects. But now, it seems possible to present at least some preliminary hypotheses and 
calculations. Let us list the phenomena recorded by us earlier for an aqueous solution of a strong electrolyte (in our case, a sodium chloride solution with a mass fraction of $0.9 \%$ or physiological solution):

- The electrical potential arising from any accelerated movement of a portion of the solution or the passage of an acoustic wave through it is measured. It should be noted here that the only known acoustoelectric effect in electrolyte solutions is the Debye ionic vibration potential (IVP) 2 27. Most likely, this is what we observe. But at low frequencies, the acoustoelectric effect has never been recorded before [6], except for an earlier study [8] where a jet was created in a solution using a piston pump, which can be thought of as a very low-frequency acoustic wave 9 .

- For the first time, the radio-frequency anisotropy of the solution was discovered, which arises owing to the propagation in it of an acoustic wave, whereas the angle of rotation of the polarization vector is proportional to the energy of the acoustic pulse and does not depend on the power of the RF signal.

- The acoustic wave passing through the solution causes a residual effect in the form of preserving the deviation of the polarization vector for a long time (not less than tens of seconds), i.e. there is a "memory" effect [10] that has never been observed before in electrolyte solutions that do not contain specifically interacting particles (liquid crystals, polymers, colloids, etc.).

Phenomena of IVP and radio-frequency anisotropy arise under the same conditions of solution disturbance, i.e. with mechanical or acoustic excitation. Therefore it would be correct, in our opinion, to try to assess the possible relations between the registered phenomena.

However, it should be taken into account that we cannot use some basic concepts: for instance, instead of considering an object as isotropic, it will be essential to introduce either the concept of orthotropy of a solution in the receiving plane (i.e. there is always anisotropy in a resting solution, and upon excitation of the solution it can change) or explain its occurrence in our experiments in some other way.

Meanwhile, it will be also needed to assume the system's invariance in time. Moreover, when considering the memory effect, one will also have to discard the hypothesis of ergodicity [11.

Let's ground the foregoing. It is known that the electrical conductivity of classical electrolyte solutions is, by definition, isotropic property that does not depend on the history of the system (within the framework of the immanent hypothesis, the deviation of the polarization angle is functionally associated with electrical conductivity). However, in the result of our experiments, two phenomena contradicting this statement were found, namely:

Rotation of the plane of polarization, i.e. the presence of a path difference between the ordinary and extraordinary waves inside the solution. If the electrical conductivity remained isotropic, any of its simultaneous (or infinitely slow) change for the entire solution would rotate the plane of polarization in no way. It seems that in our case, the electrical conductivity transforms into a tensor changing over time. Such a phenomenon, so far, has been observed either for crystals with preferential directions or for specifically oriented structures [12, 13. 
Presence of a long-term "memory" of the system. Classical physical chemistry of electrolyte solutions studies the solution in thermodynamic equilibrium. Moreover, all the integral properties of such a solution are state functions, i.e., by definition, they do not depend on the system history. To transport properties (not thermodynamic but rather kinetic, such as electrical conductivity) over long time intervals are also considered as state functions. This is an implication of the ergodicity [11] of the system, i.e. the equivalence of the average over the volume of the entire system at a certain instant of time and the average of a small part of this system over a sufficiently long time period. Simply put, if one looks at a very small piece of a system long enough, one can get an accurate depiction of the properties of the entire system. Ergodic systems, by definition, have no memory, and the average values of the properties of such systems do not depend on their prehistory.

In our experiments, nothing of that kind is observed. The system demonstrates the dependence of the properties on prehistory at macroscopic time intervals (at least tens of seconds). Such properties are more typical for polymers [14, and the description of such systems has been arousing steady interest for a long time $[10,15]$.

Obviously, the classical thermodynamic equilibrium (or close to it) description of a solution is not applicable here at all. A correct description of such a system requires the use of non-equilibrium thermodynamics. In addition, such systems radically cannot be simulated by the classical Monte Carlo method since the coordinates and momenta of particles in a non-ergodic system are correlated, and the behavior of the particles in time is not a Markov chain. Therefore, in our work, we will rely solely on experimental data and apply the required mathematical apparatus "on demand."

To simplify the problem, let's consider the process of the effects' appearance only for acoustic wave propagation in the solution since the same effects in the agitation and jet flow conditions will be described more complex.

Let's first address the appearance of the Debye potential upon low-frequency acoustic excitation of the solution, assuming the effects of electric potential generation and the rotation of the Jones vector to be somehow interrelated, and perhaps this hypothesis will help us understand the cause of the radio frequency anisotropy. It is generally accepted [6] that the Debye IVP in electrolyte solutions arises due to the difference in the inert masses of solvated cations and anions, as well as because of their different mobility in a viscous solvent.

For the first time, the IVP phenomenon was theoretically predicted by Debye in 1933 [2], who proposed an equation expressing the dependence of the electric potential on the oscillatory speed. The equation did not take into account the electrophoretic and relaxation effects, as well as the frequency dependence of the permittivity and electrical conductivity of the solution at high frequencies. Eger, Bugosh, and Govorka [4] derived an equation that takes these effects into account, and in this form, they used it to process the first experimental data on IVP at an ultrasonic frequency of 265 $\mathrm{kHz}$ 3]. It should be noted that the problem of the frequency dependence of the Debye potential in a wide frequency range has never been a subject of a special study. The authors of 5$]$ pointed out the virtual independence (NB!) of the IVP amplitude from the ultrasound frequency in the common measurement range $(100 \mathrm{kHz}-1 \mathrm{MHz})$, and this is well explained by the fact that the amplitude of the vibrational velocity does not depend on the frequency [16.

At frequencies above $100 \mathrm{MHz}$, theory [4] predicts a decrease in the amplitude of the IVP due to 
the inertness of massive ions. In the high-frequency region, the Debye potential can also be shunted by the parasitic capacitance of the solution, especially at low electrolyte concentrations [6].

In the low-frequency region, the theory predicts a constant non-zero IVP amplitude at a frequency tending to zero (NB!), what is absolutely impossible due to energy restraints as maintaining a constant potential in an electrically conductive medium requires continuous energy consumption. In other words, the ohmic resistance of the solution shunts the emerging potential at low frequencies (similar to the capacitance in the high-frequency region), what the existing theory $[4,6]$ does not take into account.

In our previous work [1], a successful attempt to register the low-frequency Debye potential was made. Here we provide a simplified diagram of our installation (see 1a). We also performed a theoretical calculation according to the formula [6]:

$$
\frac{\Phi_{0}}{\alpha_{0}}=\frac{c}{10 N_{A} e}\left[\frac{t_{+}}{z_{+}}\left(W_{+}-\frac{R T}{c^{2}}\right)-\frac{t_{-}}{z_{-}}\left(W_{-}-\frac{R T}{c^{2}}\right)\right] \frac{4 \pi L_{0}}{\omega \epsilon \sqrt{\left(\frac{\epsilon_{\omega}}{\epsilon}\right)^{2}+\left(\frac{4 \pi L_{\omega}}{\omega \epsilon}\right)^{2}}}
$$

where $\Phi_{0}$ is an amplitude of an arising potential, $\mu V ; \alpha_{0}$ - amplitude of the vibrational velocity of the solvent, $\mathrm{cm} / \mathrm{s} ; c$ - speed of sound propagation in solution, $\mathrm{cm} / \mathrm{s} ; N_{A}-$ Avogadro number, $\mathrm{mol}^{-1}$; $e$ - electron charge, $\mathrm{C} ; t_{+}, t_{-}, z_{+}, z_{-}-$transfer numbers and charge moduli of cation and anion; $W_{+}$ and $W_{-}-$apparent molar masses of the cation and anion, $\mathrm{g} / \mathrm{mol} ; \omega$ - circular vibration frequency, $\mathrm{rad} / \mathrm{s} ; L_{0}$ and $L_{\omega}$ - electrical conductivity of the solution at zero frequency and at circular frequency $\omega$, respectively, CGS-ESU; $\epsilon$ and $\epsilon_{\omega}$ - solvent permittivities at the same frequencies for a $0.9 \% \mathrm{NaCl}$ solution at a temperature of $22^{\circ} \mathrm{C}$.

The temperature and frequency dependences of the dielectric constant of the solvent, and the electrical conductivity of the solution, as well as the speeds of sound in it, required for the calculation, were taken from 17 . 19 .

Results of the calculation versus the data of our measurements are shown in the graph (1b). The predictions of the theory totally contradict our experiment in the low-frequency region, the recorded FRF of the Debye effect is very different in the low-frequency region from the theoretically estimated.

Apparently, the basic theory [4-6] did not pay much attention to low-frequency processes, the more so as the registration $[3,5]$ of low-level signals in the absence of specialized instrumental low-noise operational amplifiers at such frequencies was an issue at that time.

While the Debye potential has rapid "roll-off" at low frequencies, this effect is not observed for the $\mathrm{RF}$ polarization vector, i.e. unlike the electric potential, $\mathrm{RF}$ anisotropy behaves quite differently.

To compare the Debye process and RF anisotropy in the time domain, we used the Heaviside function as an analytical signal (see 2):

In the case of excitation in the form of a Heaviside function, the exponential decay in the Debye potential is due to ohmic shunting at low frequencies, while the explanation of the Jones vector behavior is not so obvious. Indeed, any deviation of an isotropic medium from thermodynamic equilibrium relaxes with time exponentially 20], and one could consequently expect the Jones vector, like the Debye potential, would be asymptotically approaching the initial position over time. However, 


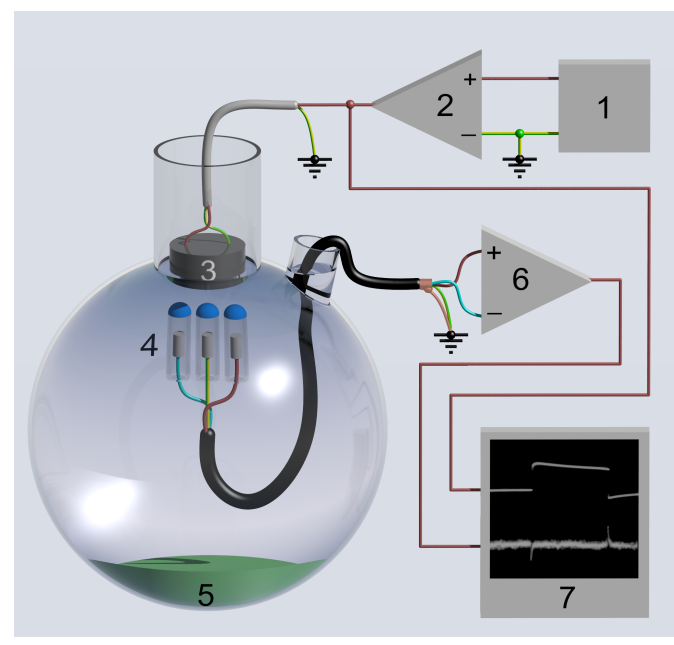

(a)

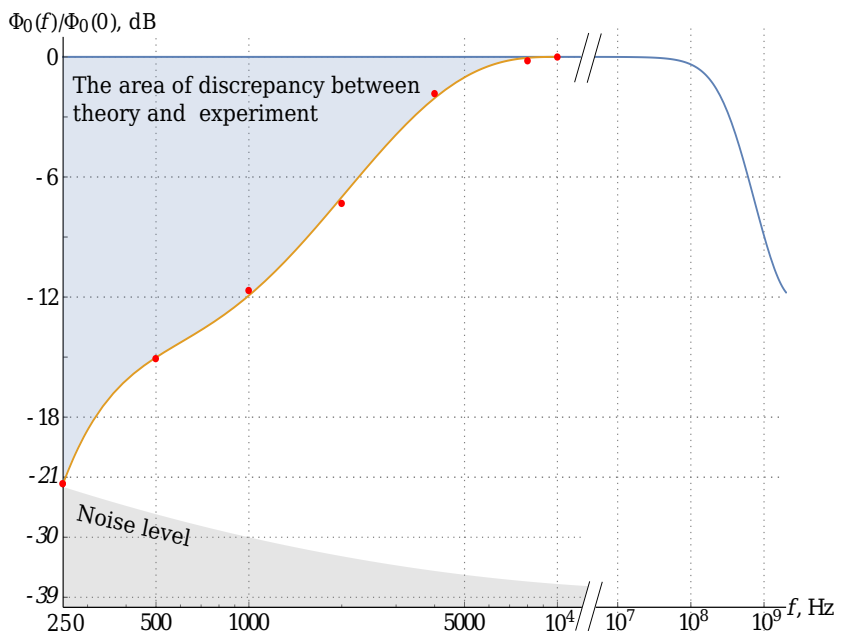

(b)

Figure 1: (a) Installation for acoustoelectric effect registration. 1 - generator; 2 - power amplifier; 3 - emitter; 4 - silver chloride electrodes under protective caps; 5 - sound-absorbing cover; 6 instrumental OA; 7 - recorder. (b) FRF of the recorded signal. Red dots - experimental results, blue curve - calculated data for $0.9 \% \mathrm{NaCl}$ solution at $22^{\circ} \mathrm{C}$ in accordance with the basic theory [4, $6]$

instead of this, "memorizing" of the position of the maximum deviation happens for some time (on the order of units/tens of seconds), followed by normal relaxation. Therefore, in the very mechanism of the solution anisotropy change, there must be an integration process, or rather, something similar to an analog sample and hold circuit.

Let's take that no chemical or physical processes in the solution add "external" energy [1], and its only source is an acoustic wave. At the same time, as an initial condition, it is necessary to exclude the hypothesis of rotation of the polarization vector under the influence of any electric potential applied to the solution, the Debye potential in particular. For this, we carried out experiments with passing an electric current through the solution in the frequency band from $0 \mathrm{~Hz}$ to $10 \mathrm{kHz}$, and voltages from 0 to $2 \mathrm{~V}$. No of them caused any deviations of the RF polarization vector, though the signal level in the experiment exceeded the Debye potential recorded on the same setup (1b) by $116 \mathrm{~dB}$. This allows us to make an unambiguous conclusion: the polarization vector of the radiofrequency signal passing through the solution does not rotate under the influence of an applied voltage. Thus, we can accept that the "electric part" of the Debye effect cannot be the root of the radio frequency anisotropy; it would be more correct to assume that the mechanism that "triggers" the Debye effect can also trigger the rotation of the RF signal polarization.

The system relaxation after the acoustic wavefront passage can be modeled by an ideal differentiating circuit. The Debye process (given its experimental frequency response recorded by us in the low-frequency region) can also be described in the time domain by a differentiating circuit.

We also assume that the power flux density of the process " $\mathrm{X}$ ", responsible both for the rotation of the polarization vector, is proportional to the square of Debye electric potential voltage (considering no significant reactive component in the load). Then the process model can be represented in the 

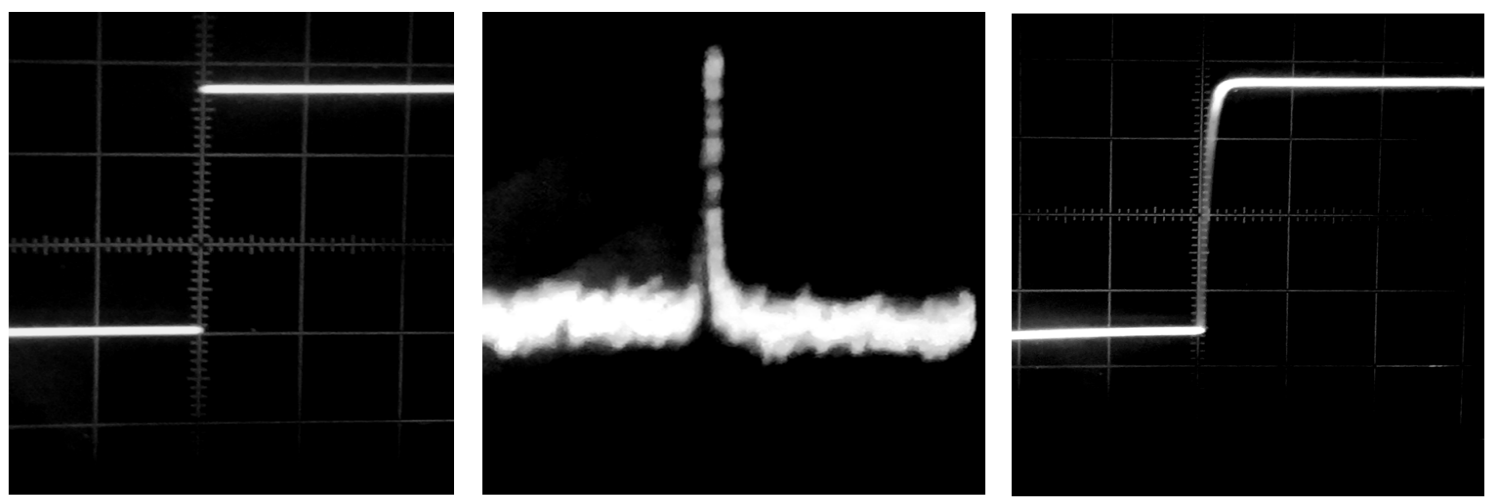

Figure 2: On the left - the Heaviside signal fed to the hydroacoustic emitter, in the center - the Debye potential $\mathrm{U}=20 \mu \mathrm{V}$, on the right - the rotation of the Jones vector (all images are captured from the oscilloscope screen)

form of the following electrical circuit (3).

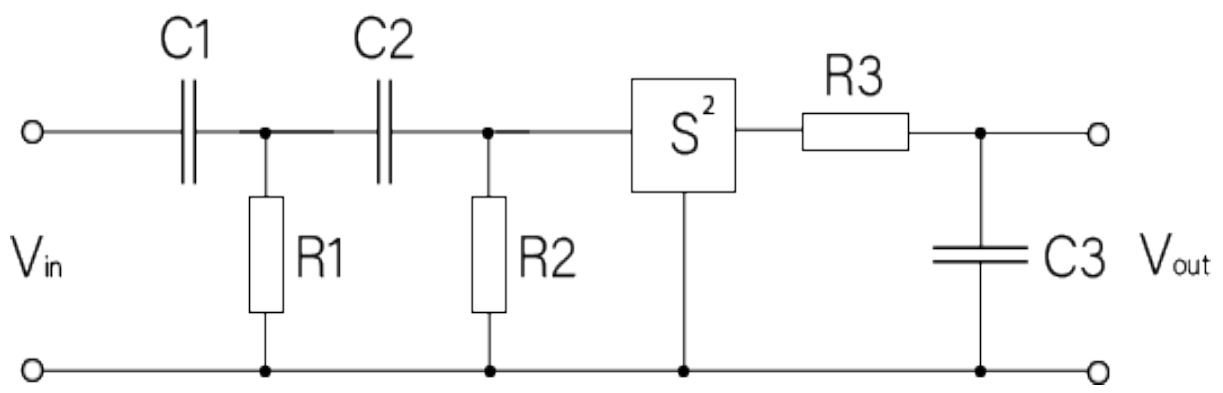

Figure 3: The simplified electrical circuit of the proposed model of the process. The $C_{1} R_{1}$ circuit simulates the energy dissipation of an acoustic wave, the $C_{2} R_{2}$ circuit - lower band limitation for the Debye effect, $S^{2}$ - a square-law generator, the $C_{3} R_{3}$ circuit - a hypothetical integrator, which physical nature is under investigation

Of course, the transfer function of ideal differentiating circuits does not satisfy the conditions of physical feasibility [21]. However, in our case, the energy of the acoustic wave is finite by definition what makes it possible to use ideal circuit as a model. If $\tau_{1}$ is determined by (R1, C1) and $\tau_{2}$ is determined by $(\mathrm{R} 2, \mathrm{C} 2)$, then the analytical solution of this model can be derived for the angle of the polarization deviation $\Delta \alpha$ as follows:

$$
\Delta \alpha \simeq \int_{0}^{T}\left(e^{-\frac{t}{\tau_{1}}} \cdot e^{-\frac{t}{\tau_{2}}}\right)^{2} d t=\int_{0}^{T} e^{-2 \frac{t^{2}}{\tau_{1} \tau_{2}}} d t
$$

Let's introduce a new variable $z=t \cdot\left(\frac{2}{\tau_{1} \tau_{2}}\right)^{\frac{1}{2}}$ so that $d t=\left(\frac{\tau_{1} \tau_{2}}{2}\right)^{\frac{1}{2}} d z$. For convenience we'll define multiplier $\left(\frac{2}{\tau_{1} \tau_{2}}\right)^{\frac{1}{2}}$ as a constant $k$, then the original integral takes the following form

$$
\frac{1}{k} \int_{0}^{k T} e^{-z^{2}} d z
$$


First we accept that $k T>>1$, then we can estimate the value of this integral by the so-called large parameter method. We represent the integral on $\mathrm{z}$ as the difference of two integrals, i.e. the first will be equal to half of the Gaussian tabular integral, and the second will be integrated by parts (IBP).

$$
\int_{0}^{k T} e^{-z^{2}} d z=\int_{0}^{\infty} e^{-z^{2}} d z-\int_{k T}^{\infty} e^{-z^{2}} d z=\frac{\sqrt{\pi}}{2}-\int_{k T}^{\infty} e^{-z^{2}} d z
$$

To calculate the second integral by the IBP, we introduce a new variable $\zeta=z^{2}$ so that $d z=\frac{d \zeta}{2 \sqrt{\zeta}}$ and

$$
\int_{k T}^{\infty} e^{-z^{2}} d z=\int_{(k T)^{2}}^{\infty} \frac{1}{2 \zeta^{\frac{1}{2}}} e^{-\zeta} d \zeta
$$

. Integration by parts is according to the following rule:

$$
\int u d v=u v-\int v d u
$$

Let $u=\frac{1}{2 \zeta^{\frac{1}{2}}}$ and $d u=-\frac{1}{4 \zeta^{\frac{3}{2}}}$ then $d v=e^{-\zeta} d \zeta$ and $v=\int d v=-e^{-\zeta}$, and the original integral takes the form:

$$
-\left.\frac{1}{2 \zeta^{\frac{1}{2}}} e^{-\zeta}\right|_{(k T)^{2}} ^{\infty}-\int_{(k T)^{2}}^{\infty} \frac{1}{4 \zeta^{\frac{3}{2}}} e^{-\zeta} d \zeta
$$

Notable that the power of $\zeta$ under the integral sigh has increased, i.e. $\int_{(k T)^{2}}^{\infty} \frac{1}{4 \zeta^{\frac{3}{2}}} e^{-\zeta} d \zeta<\int_{(k T)^{2}}^{\infty} \frac{1}{2 \zeta^{\frac{1}{2}}} e^{-\zeta}$. Let's make sure that each next term of the expression is less than the previous one:

$$
\begin{gathered}
-\left.\frac{1}{2 \zeta^{\frac{1}{2}}} e^{-\zeta}\right|_{(k T)^{2}} ^{\infty}-\int_{(k T)^{2}}^{\infty} \frac{1}{4 \zeta^{\frac{3}{2}}} e^{-\zeta} d \zeta=-\left.\frac{1}{2 \zeta^{\frac{1}{2}}} e^{-\zeta}\right|_{(k T)^{2}} ^{\infty}+\left.\frac{1}{4 \zeta^{\frac{3}{2}}} e^{-\zeta}\right|_{(k T)^{2}} ^{\infty}-\int_{(k T)^{2}}^{\infty} \frac{3}{8 \zeta^{\frac{5}{2}}} e^{-\zeta} d \zeta= \\
=-\left.\frac{1}{2 \zeta^{\frac{1}{2}}} e^{-\zeta}\right|_{(k T)^{2}} ^{\infty}+\left.\frac{1}{4 \zeta^{\frac{3}{2}}} e^{-\zeta}\right|_{(k T)^{2}} ^{\infty}-\left.\frac{3}{8 \zeta^{\frac{5}{2}}} e^{-\zeta}\right|_{(k T)^{2}} ^{\infty}+\ldots
\end{gathered}
$$

Let's go back to the variable z:

$$
\int_{k T}^{\infty} e^{-z^{2}} d z=-\left.\frac{1}{2 z^{\frac{1}{2}}} e^{-z}\right|_{k T} ^{\infty}+\left.\frac{1}{4 z^{3}} e^{-z^{2}}\right|_{k T} ^{\infty}-\left.\frac{3}{8 z^{5}} e^{-z^{2}}\right|_{k T} ^{\infty}+\ldots
$$

The general term of the series obtained with IBP can be written as

$$
a_{n}=\frac{(-1)^{n} \cdot(2 n-1) ! !}{2^{n+1} \cdot z^{2 n+1}}
$$

A series with such a general term converges only up to some $\mathrm{N}$, depending on the parameter of the original integral. This is an example of an asymptotic series: the series approximates the original integral by the sum of the first $\mathrm{N}$ terms, such that the double factorial in the numerator remains less than the denominator.

Let's return to the variable of integration $t$ and write down the corresponding expression of the 
series:

$$
\sum_{n=0}^{N} a_{n}=-\left.e^{\frac{-2 t^{2}}{\tau_{1} \tau_{2}}} \cdot \sum_{n=0}^{N} \frac{(-1)^{n} \cdot(2 n-1) ! !}{2^{n+1} \cdot\left(t \cdot \sqrt{\frac{2}{\tau_{1} \tau_{2}}}\right)^{2 n+1}}\right|_{T} ^{\infty}
$$

Finally, we can write down the expression for our target value $\Delta \alpha$ and find its value within the limits of integration:

$$
\begin{aligned}
& \Delta \alpha \simeq \sqrt{\frac{\tau_{1} \tau_{2}}{2}} \cdot\left[\frac{\sqrt{\pi}}{2}+\left.e^{\frac{-2 t^{2}}{\tau_{1} \tau_{2}}} \cdot \sum_{n=0}^{N} \frac{(-1)^{n} \cdot(2 n-1) ! !}{2^{n+1} \cdot\left(t \cdot \sqrt{\frac{2}{\tau_{1} \tau_{2}}}\right)^{2 n+1}}\right|_{T} ^{\infty}\right]= \\
& \sqrt{\frac{\tau_{1} \tau_{2}}{2}} \cdot\left[\frac{\sqrt{\pi}}{2}-e^{\frac{-2 T^{2}}{\tau_{1} \tau_{2}}} \cdot \sum_{n=0}^{N} \frac{(-1)^{n} \cdot(2 n-1) ! !}{2^{n+1} \cdot\left(T \cdot \sqrt{\frac{2}{\tau_{1} \tau_{2}}}\right)^{2 n+1}}\right]
\end{aligned}
$$

Let's also check if the model is physically feasible and stable. To do this we will use the Laplace transform. In our model, the complex transfer function of the series-connected cascades is equal to the product of the complex transfer functions of all the in-series cascades; in our case, this means $H(s)=H_{1}(s) \cdot H_{2}(s) \cdot H_{3}(s)$.

Let's look at the transfer functions of each of the three introduced four-poles below. EMF source $\epsilon(t)$ is connected to the input of the first four-pole. Since the resistance of the capacitor is reactive, let's move to the frequency domain and write the equation for the first circuit:

$$
E(j \omega)=I(j \omega) \cdot\left(R_{1}+\frac{1}{j \omega C_{1}}\right)
$$

Here and in the following, it is convenient to introduce a complex variable with zero real part $s=j \omega$.

The complex transfer function is defined as the ratio of the system output to its input: $H(s)=$ $\frac{X_{o} u t(s)}{X_{i} n(s)}$. In the first circuit under consideration, the input is the voltage $E(s)$, and the output is the voltage $U_{1}$ across the resistor $R_{1}$. Let's find this voltage, it is equal to the product of the current $I(s)$ by the resistance $R_{1}$. The current $I(s)$, in turn, is defined as the ratio of the EMF $E(s)$ to the total resistance of the circuit, i.e.

$$
I(s)=\frac{E(s)}{R_{1}+\frac{1}{s C_{1}}}
$$

Accordingly, the output voltage of the system

$$
U_{1}=I(s) \cdot R_{1}=\frac{E(s) R_{1}}{R_{1}+\frac{1}{s C_{1}}}
$$

Coming back to the definition of the complex transfer function, we obtain

$$
H_{1}=\frac{E(s)}{U_{1}(s)}=\frac{R_{1}}{R_{1}+\frac{1}{s C_{1}}}
$$

Analysis of the two next four-poles shares the same insights, except for the fact that there's a 
transition from instantaneous voltage to power in the third cascade, i.e.

$$
\begin{gathered}
H_{2}=\frac{U_{1}(s)}{U_{2}(s)}=\frac{R_{2}}{R_{2}+\frac{1}{s C_{2}}} \\
H_{3}=\frac{P_{2}(s)}{P_{3}(s)}=\frac{1 / s C_{3}}{R_{3}+1 / s C_{3}}
\end{gathered}
$$

Owning definitions of all three transfer functions, let's write the expression for the entire system:

$$
H=\frac{R_{1}}{R_{1}+1 / s C_{1}} \cdot \frac{R_{2}}{R_{2}+1 / s C_{2}} \cdot \frac{1 / s C_{3}}{R_{3}+1 / s C_{3}}
$$

For the subsequent reduction of similar ones, it is convenient to introduce a new constant $\tau=R C$ the time constant of the RC circuit.

$$
\begin{gathered}
H=\frac{R_{1} \cdot R_{2}}{\frac{\left(1+\tau_{1} s\right) \cdot\left(1+\tau_{2} s\right) \cdot\left(1+\tau_{3} s\right)}{C_{1} C_{2} s^{2}}}=\frac{\tau_{1} \tau_{2} \cdot s^{2}}{\left(1+\tau_{1} s\right) \cdot\left(1+\tau_{2} s\right) \cdot\left(1+\tau_{3} s\right)}=\frac{\tau_{1} \tau_{2} \cdot s^{2}}{\left(1+\tau_{2} s+\tau_{1} s+\tau_{1} \tau_{2} s^{2}\right) \cdot\left(1+\tau_{3} s\right)}= \\
=\frac{\tau_{1} \tau_{2} \cdot s^{2}}{1+\tau_{2} s+\tau_{1} s+\tau_{1} \tau_{2} s^{2}+\tau_{3} s+\tau_{2} \tau_{3} s^{2}+\tau_{1} \tau_{3} s^{2}+\tau_{1} \tau_{2} \tau_{3} s^{3}}= \\
=\frac{s^{2} \cdot \tau_{1} \tau_{2}}{1+s\left(\tau_{1}+\tau_{2}+\tau_{3}\right)+s^{2}\left(\tau_{1} \tau_{2}+\tau_{1} \tau_{3}+\tau_{2} \tau_{3}\right)+s^{3} \cdot \tau_{1} \tau_{2} \tau_{3}}
\end{gathered}
$$

Since the given circuit is a modeling one, the values of resistances and capacitances cannot be absolutely random, namely it is necessary to adhere to the following relations over the numerical calculation of the transfer function: $C_{1}>>C_{2}>>C_{3} ; R_{3}>>R_{2}>>R_{1}$.

Let's take the following values for example: $R_{1}=1 \mathrm{Ohm}, R_{2}=100 \mathrm{Ohm}, R_{3}=10 \mathrm{kOhm}$, $C_{3}=50 \mu \mathrm{F}, C_{2}=0.5 \mathrm{mF}, C_{1}=50 \mathrm{mF}$. The poles of the transfer function (on the complex $s$-plane) are located at point $(-2)$ on the real axis and have a zero imaginary coordinate, while its zero is at the origin point (4).

Now we can argue that the model is physically feasible, stable 22, and ready to work. Let's check the results of a software simulation on the system response to the Heaviside signal. Let's look in the time domain at the original signal, the Debye potential, and the rotation of the RF signal polarization vector 5 .

As one can see, software simulation provides a satisfactory fit between the real and waveforms synthesized by the model. The negligible difference is due to the not quite accurate selection of the time parameters, which was carried out, of course, empirically.

It is clear that analogy is not proof, so for now, only a provident conclusion can be drawn: some physical process "X", which has properties of an integrating circuit, can transform the energy of an acoustic signal in such a way that the radio-frequency polarization vector changes its position and remembers it to some, quite significant, time.

The physical structure of this integrator cannot be electrical or molecular for the reasons stated earlier (in [1] as well). Then, according to the principle of exclusion, it would be acceptable to assume the gyroscopic mechanism of the observed effect. 


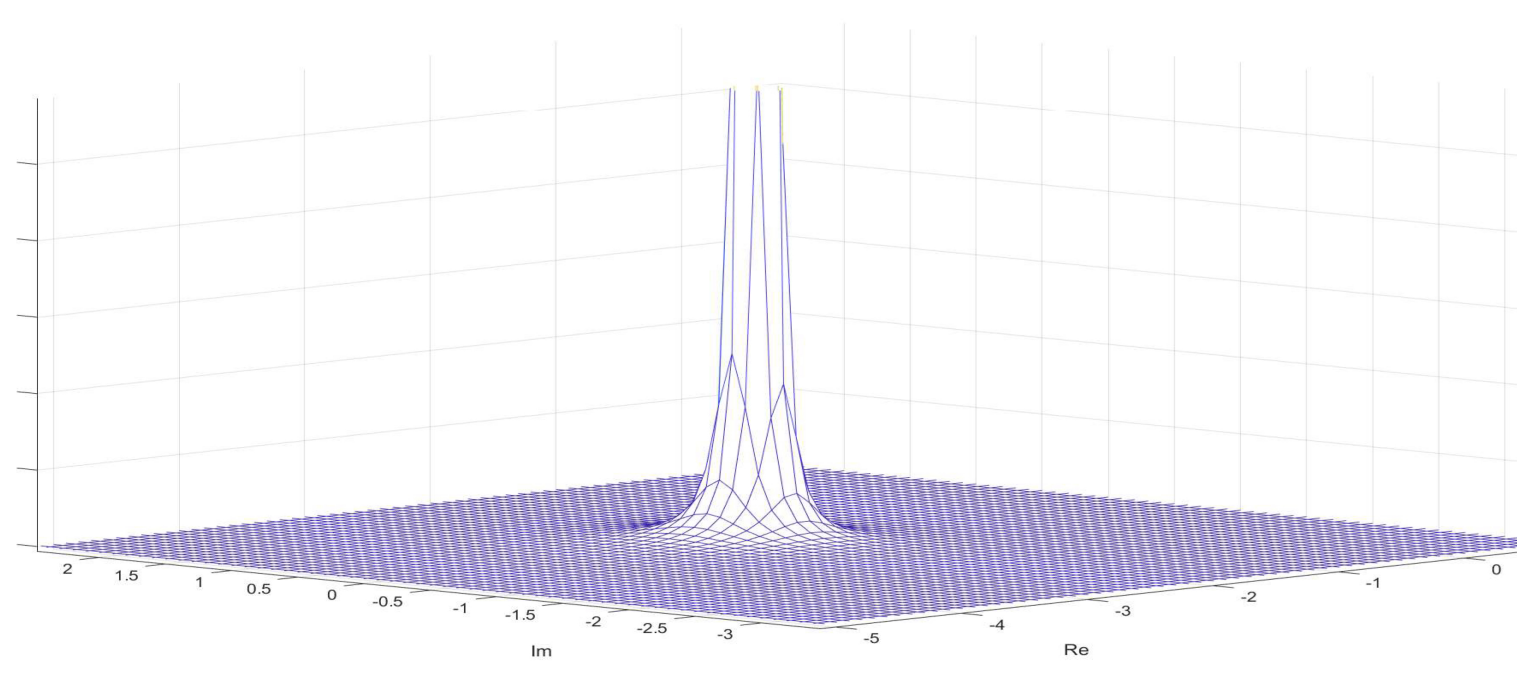

Figure 4: Complex s-plane mapping of the signal

Indeed, if the pendulum gyrocompass is placed in a gravitational field for a moment, then it'll start to change the orientation of the angular momentum vector. If in a moment we "turn off gravitation", then the gyrocompass will perform the functions of a gyroscope, i.e. hold the position of the rotation axis 23. According to the principle of equivalence, if we replace gravity with the corresponding acceleration, then the gyrocompass will behave in the same way.

Liquid gyroscopes utilizing acidified water or aqueous solutions of lithium salts as a working medium are well known 24, it is also known that any vibrations (NB!) are an undesirable effect for them, but the reports on experiments propagating an acoustic wave through the working medium were not found by us.

However, a precondition of such gyroscopes operation is a radio-frequency field, which presence leads to the precession of the working body nuclei. In our case, however, the effect doesn't depend on the field strength, i.e. we have to assume that the rotation of the RF signal polarization vector is due to a change in either the electronic moment of the atoms or the magnetic moment of the nuclei of ions with an odd mass number. How an acoustic wave can affect the rotation of the magnetic moment carriers is yet unclear for us; large-scale experimental and theoretical studies are absolutely necessary.

So, based on the simulation results, we assume that the discussed process " $\mathrm{X}$ " can consistently fit into the framework of known physical effects. An additional argument in favor of the "gyroscopic" hypothesis may be the "saturation effect" which was discovered in our experimental work as the radio-frequency polarization vector under the influence of the acoustic wave was shifted at a certain angle increasing less and less whereas the wave amplitude rises, and the process of polarization vector deviation never turned into rotation.

It can also be assumed that the changing of the electronic and/or nuclear moment orientation under the influence of external acceleration always occurs in water, and electrolyte ions are needed 

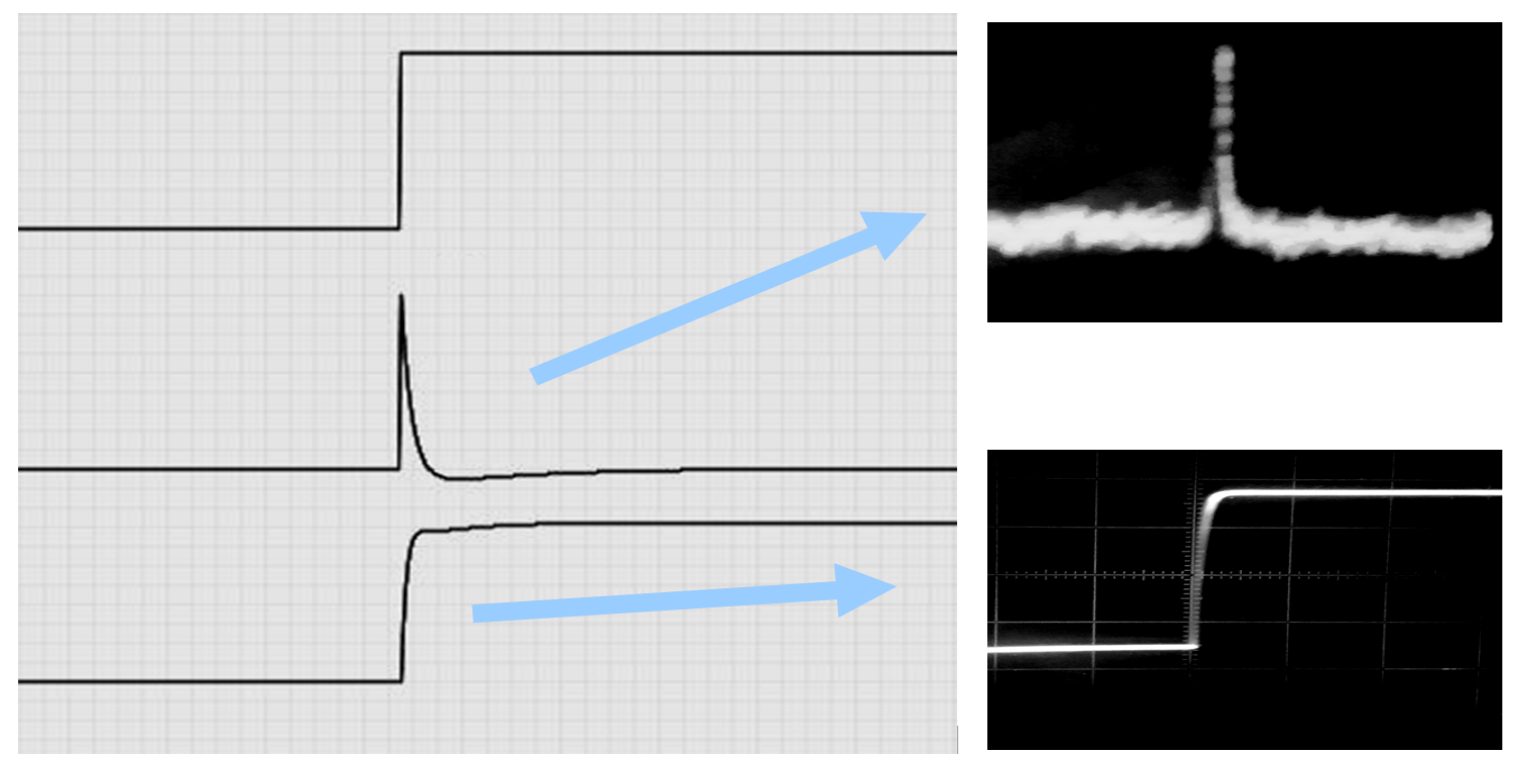

Figure 5: The upper curve is the Heaviside signal, in the middle - Debye potential, in the bottom rotation of the RF signal polarization vector. Real experimental curves are shown on the right for comparison.

only to register the deviation of the polarization vector in the radio frequency range. If this assumption is faithful, then it will be necessary to re-examine numerous existing ideas about the behavior of aquatic environments. For example, the physics of the "wake of a submarine" detected by radars 25], the biophysical mechanism of cardiac electrical signals generation [8], and many other phenomena.

Implicitly that laboratory equipment and adequate working conditions are required to continue work in this direction and obtain accurate prooving, what is not the authors' case. Nevertheless, some promising engineering devices (in addition to those described in the previous paper [1]) are likely. E.g. it was found that the anisotropy of the solution is transported by the carrier (Sic!), i.e. if one pumps the solution which changed the polarization vector under acoustic disturbance, then the trace of the disturbance is preserved. This phenomenon makes it possible to develop a completely contactless unitary sensor of velocity and liquid inhomogeneities (the experimental model has already shown the feasibility of such an idea even for tap water).

\section{References}

[1] A. V. Kramarenko, A. V. Kramarenko, and O. Savenko, "A new radio-frequency acoustic method for remote study of liquids," Scientific Reports, vol. 11, p. 6696, 2021. DOI: 10.1038/ s41598-021-84500-6.

[2] P. Debye, "A method for the determination of the mass of electrolytic ions," The Journal of Chemical Physics, vol. 1, pp. 13-16, 1933. DOI: 10.1063/1.1749213. 
[3] E. Yeager, J. Bugosh, F. Hovorka, and J. McCarthy, "The application of ultrasonic waves to the study of electrolytic solutions. II. The detection of the debye effect," The Journal of Chemical Physics, vol. 17, pp. 411-415, 1949. DOI: 10.1063/1.1747269.

[4] J. Bugosh, E. Yeager, and F. Hovorka, "The application of ultrasonic waves to the study of electrolytic solutions. I. A modification of debye's equation for the determination of the masses of electrolytic ions by means of ultrasonic waves," The Journal of Chemical Physics, vol. 15, no. 8, pp. 592-597, 1947. DOI: 10.1063/1.1746602. [Online]. Available: https : //doi.org/10.1063/1.1746602.

[5] R. Zana and E. Yeager, "Ultrasonic vibration potentials and their use in the determination of ionic partial molal volumes," The Journal of Physical Chemistry, vol. 71, pp. 521-536, 1967.

[6] R. Zanaf and E. B. Yeager, "Ultrasonic vibration potentials," Modern Aspects of Electrochemistry, pp. 1-60, 1982.

[7] W. Shougang, K. N. Cuong, and J. D. Gerald, Ultrasonic vibration potential imaging: theory and experiments. VDM Verlag Dr. Müller, 2010, IsBN: 9783639241570.

[8] A. V. Kramarenko, G. P. Kulemin, and A. M. Savchuk, "Gemodinamicheskiǔ komponent élektrokardiogrammy," Biofizika, vol. 41, pp. 686-694, 1996.

[9] J. Lighthill, Waves in fluids, ser. Cambridge mathematical library. Cambridge University Press, 2001.

[10] B. D. Coleman, "Thermodynamics of materials with memory," Archive for Rational Mechanics and Analysis, vol. 17, pp. 1-46, 1964.

[11] M. Brin and G. Stuck, Introduction to dynamical systems. Cambridge University Press, 1964.

[12] B. Y. Balagurov, "Conduction of the three-dimensional model of a composite with structural anisotropy," Journal of Experimental and Theoretical Physics, vol. 123, pp. 348-356, 2016.

[13] B. T. Kolomiets, V. M. Lyubin, and V. P. Shilo, "Anizotropization of the electric conductivity in oriented samples of vitreous semiconductors," Journal of Experimental and Theoretical Physics Letters, vol. 17, p. 412, 1973.

[14] G. Astarita, "Thermodynamics and relaxation. the relationship between memory for past history and equilibrium," Journal of Membrane Science, vol. 3, pp. 163-178, 1978.

[15] B. D. Coleman and V. J. Mizel, "On thermodynamic conditions for the stability of evolving systems," Archive for Rational Mechanics and Analysis, vol. 29, pp. 105-113, 1968.

[16] D. T. Blackstock, Fundamentals of physical acoustics. Nashville, TN: John Wiley \& Sons, 2000.

[17] H. J. Liebe, G. A. Hufford, and T. Manabe, "A model for the complex permittivity of water at frequencies below 1 thz," International Journal of Infrared and Millimeter Waves, vol. 12, pp. 659-675, 1991.

[18] S. A., "Equations for calculating the dielectric constant of saline water (correspondence)," IEEE Transactions on Microwave Theory and Techniques, vol. 19, pp. 733-736, 1971. 
[19] S. J. Kleis and L. A. Sanchez, "Dependence of speed of sound on salinity and temperature in concentrated $\mathrm{NaCl}$ solutions," Solar Energy, vol. 45, pp. 201-206, 1990.

[20] D. J. Evans, D. J. Searles, and S. R. Williams, "Dissipation and the relaxation to equilibrium," Journal of Statistical Mechanics: Theory and Experiment, vol. 2009, P07029, 2009.

[21] C. K. Alexander and M. N. O. Sadiku, Capacitors and inductors, ser. Fundamentals of electric circuits. McGraw Hill, 2013, ISBN: 9783030024055.

[22] A. Bacciotti, Stability and Control of Linear Systems, ser. Studies in Systems, Decision and Control. Springer International Publishing, 2018, ISBN: 9783030024055.

[23] N. Ivanovsky, L. Kozachenko, and A. Ivanov, Technical means of navigation. Kerchen State Maritime Technological University, 2020.

[24] R. M. Umarkhodzhaev, Y. V. Pavlov, and A. N. Vasil'ev, "Istoriya razrabotki hiroskopa na osnove yadernoho mahnitnoho rezonansa v rossii v 1960-2000-e hody," Hiroskopiya i navihatsiya, pp. 3-27, 2018.

[25] M. Zhang and J. Wang, "Microwave scattering from submerged object induced wake over rough sea surface," 2017 XXXIInd General Assembly and Scientific Symposium of the International Union of Radio Scie pp. 1-4, 2017. 